\title{
Evolutionary Squeaky Wheel Optimization: A New Framework for Analysis
}

\author{
Jingpeng Li \\ School of Computer Science, The University of Nottingham, Nottingham, \\ NG8 1BB, United Kingdom
}

Andrew J. Parkes

School of Computer Science, The University of Nottingham, Nottingham, NG8 1BB, United Kingdom

Edmund K. Burke

ekb@cs.nott.ac.uk

School of Computer Science, The University of Nottingham, Nottingham,

NG8 1BB, United Kingdom

\begin{abstract}
Squeaky wheel optimization (SWO) is a relatively new metaheuristic that has been shown to be effective for many real-world problems. At each iteration SWO does a complete construction of a solution starting from the empty assignment. Although the construction uses information from previous iterations, the complete rebuilding does mean that SWO is generally effective at diversification but can suffer from a relatively weak intensification. Evolutionary SWO (ESWO) is a recent extension to SWO that is designed to improve the intensification by keeping the good components of solutions and only using SWO to reconstruct other poorer components of the solution. In such algorithms a standard challenge is to understand how the various parameters affect the search process. In order to support the future study of such issues, we propose a formal framework for the analysis of ESWO. The framework is based on Markov chains, and the main novelty arises because ESWO moves through the space of partial assignments. This makes it significantly different from the analyses used in local search (such as simulated annealing) which only move through complete assignments. Generally, the exact details of ESWO will depend on various heuristics; so we focus our approach on a case of ESWO that we call ESWO-II and that has probabilistic as opposed to heuristic selection and construction operators. For ESWO-II, we study a simple problem instance and explicitly compute the stationary distribution probability over the states of the search space. We find interesting properties of the distribution. In particular, we find that the probabilities of states generally, but not always, increase with their fitness. This nonmonotonocity is quite different from the monotonicity expected in algorithms such as simulated annealing.
\end{abstract}

\section{Keywords}

Combinatorial optimization, metaheuristics, stochastic search, stochastic process, Markov chain.

\section{Introduction}

According to Papadimitriou and Steiglitz (1982), a combinatorial problem can be expressed as a model $\Phi=(S, \Omega, f)$, where $S$ denotes a search space over a finite set of 
discrete decision variables, $\Omega$ denotes a set of constraints among the variables, and $f$ denotes an objective function $f: S \rightarrow Z^{+}$to be maximized (or minimized). Many such problems arising in various fields (such as computer science, management, engineering, etc.) cannot be solved exactly within reasonable time limits for problem instance sizes of practical interest. Heuristics attempt to achieve a trade-off between solution quality and search completeness. Metaheuristic approaches have been widely studied (Gendreau and Potvin, 2010) and can be applied, with suitable modifications, to a broad class of combinatorial optimization problems. Some well-known examples of metaheuristics are ant colony optimization (Dorigo et al., 1996), estimation of distribution algorithm (Larrañaga and Lozano, 2002), stochastic local search (for a review, see Hoos and Stutzle, 2005), genetic algorithms (Goldberg, 1989), GRASP (Feo and Resende, 1989), simulated annealing (Kirkpatrick et al., 1983), TABU search (Glover, 1989), and variable neighborhood search (Hansen and Mladenović, 1999). Based on their method for building new solutions, metaheuristics can be roughly grouped into the following two classes: constructive metaheuristics and iterative metaheuristics. The former generates a solution from scratch by successive addition of certain components (with or without backtracking), whilst the latter starts with a complete solution and changes this solution in an iterative process in order to improve the objective function value.

Among many metaheuristics reported in recent years, squeaky wheel optimization (SWO; Joslin and Clements, 1999) is relatively little known but is receiving increased attention in the research community. This method is based on the observation that in real world combinatorial problems, the solutions consist of components which are intricately woven together in a nonlinear, nonadditive fashion. To deal with these components, Joslin and Clements (1999) proposed the original idea of SWO, and applied it to production line scheduling problems and graph coloring problems with some satisfactory results. Since then, SWO has been successfully applied to many different types of discrete optimization problems, such as game playing of contract bridge (Ginsberg, 2001) air force space-ground communications (Barbulescu et al., 2004), examination time-tabling (Burke and Newall, 2004), crane scheduling (Lim et al., 2004), port space allocation (Fu et al., 2007), bandwidth multicoloring (Malaguti and Toth, 2008), machine scheduling (Feng and Lau, 2008), and two dimensional strip packing (Burke et al., 2010).

In SWO, an initial solution is first constructed by a greedy algorithm. The solution is then analyzed to assign blame to the components which cause trouble in the solution, and this information is used to modify the priority order in which the greedy algorithm constructs the new solutions. This construction-analysis-prioritization cycle continues until a stopping condition is reached. Hence, the SWO cycle has the consequence that problem components that are hard to handle tend to rise in the priority queue, and components that are easy to handle tend to sink. In essence, this method finds good quality solutions by searching in two spaces simultaneously: the traditional solution space and the new priority space.

Being a constructive metaheuristic, the SWO, however, has its own disadvantages, such as poor scalability due to the random starting point of the greedy constructor and slow convergence due to the inability to make small moves in the solution space. If its construction process only started from a partial solution for each cycle, the SWO would speed up significantly. In addition, if it were possible to restrict changes of components to the troublemakers only, the changes in the corresponding solutions would be relatively small. To address these issues, Aickelin et al. (2009) proposed an evolutionary version of SWO, and reported significantly better results than those of the original SWO 
on two manpower scheduling problems: driver scheduling ( $\mathrm{Li}$ and Kwan, 2003) and nurse scheduling (Aickelin and Li, 2007). To achieve this, evolutionary SWO (ESWO) incorporates two additional operators into the cycle: Selection and mutation. The selection operator determines whether a component should be discarded and placed in a queue for the new allocation, based on the local fitness of this component. The mutation step further discards some randomly selected components.

We also note that apart from introducing the selection and mutation operators, ESWO did not include the notion from SWO of the sorting of the constructor's priority queue (PQ) being sticky. Specifically, in SWO, the priority queue is not totally rebuilt at each iteration, but rather it uses the analysis stage to modify the PQ so as to form a new PQ that still preserves some memory of the previous PQ. For example, the items in the PQ might be limited in how far they move within the queue. In contrast, in ESWO, the constructor does not use the results of the previous iteration other than the state that it gives. This means that, although sharing some common motivations, it is rather a different algorithm from SWO. It is perhaps more akin to the many metaheuristics that use some form of iterative repair that identify flaws in a solution, and then attempt to repair them. However, the lack of memory of the previous PQ also means that it is more straightforward to build a Markov chain model based on the search space, and without having to also include the PQ as would (presumably) be necessary to model SWO.

At this point, we explore the motivations to set up studies of the properties of the algorithm. We are inspired, of course, by the numerous Markov Chain analyses of simulated annealing, SA (Michiels et al., 2007). On the technical side, the formulation itself differs from such analyses of SA because ESWO moves through states that are only partial assignments, that is, not all the variables are assigned values. In contrast, local search methods such as SA deal only with states corresponding to complete assignments; all variables are assigned values. However, we note that an increasing number of algorithms do have such movement through the space of partial assignments and thus this class of formulations might well be of increasing interest.

The practical motivation for a formal framework and (semi-empirical) analysis is to be able to better understand the interplay of the various stages within an ESWO algorithm. This is perhaps even more important than for SA, because ESWO is a multistage algorithm with potentially many parameters, and it is far from understood how the stages interact with each other.

A challenge in setting up a framework and analysis is that in practice ESWO often contains greedy heuristics with some random factors (e.g., breaking ties randomly). To overcome this drawback, in this paper, we study a particular version of ESWO, that we call ESWO-II, and that is set up to capture (as much as possible) the intent of ESWO, but to do so using stochastic methods that are susceptible to Markov chain analysis. For example, for ESWO-II, we revised the construction step to make probabilistic choices among various possible destination states, though again with a bias toward fitter solutions.

Hence, the aim of the present paper is to define ESWO-II and create an associated explicit Markov chain formulation. We then verify that it has the expected global convergence properties. For example, we will confirm that, starting from any initial solution, the ESWO-II visits the global optimal set with probability 1 (though note that it does not converge to it). The majority of the formalism will also potentially apply to general ESWO, though it would need some further modeling to capture the domain specific heuristics that it would generally include. 
Using a small but concrete example, we then make explicit calculations of the resulting probability distributions over the states. We find that the distributions could be concentrated on the good solutions-this is expected as it would probably not be a good search method otherwise. However, we do find the slightly surprising result that the probability need not vary monotonically with the fitness; sometimes states with slightly lower fitness can be more probable than those with higher fitness. This is in stark contrast to standard methods such as simulated annealing in which the equilibrium (metropolis) distribution is monotonic with respect to fitness. This property of ESWO-II might be regarded as strange, but need not be a bad sign for its effectiveness: After all, it is hitting times that matter more than final stationary distributions. The interaction between such stationary distributions and the hitting times might well be an interesting topic for future research.

Our general motivation for carrying out this study is that the Markov chain numerical solution of smaller instances can allow much faster and more precise calculation than simulation would give. This then enables a more effective study aimed at understanding how the various components within the algorithms interact with key measures such as stationary distributions. That is, the goal is to explore and understand the interactions of the various components within the search algorithm(s).

The remainder of the paper is structured as follows. Section 2 gives a short introduction into the key concepts of finite Markov chains. Section 3 elaborates a formal framework of the ESWO-II, from the state transition point of view. Section 4 presents some mathematical convergence results, and Section 5 discusses some results on stationary distribution. Section 6 contains concluding remarks and some possible future work.

\section{Standard Preliminaries of Finite Markov Chains}

In this section, we summarize various standard definitions and results that we will need later. A Markov chain is a stochastic process with the Markov property (Tijms, 2003). Being a stochastic process means that state transitions are probabilistic. The Markov property means that the present state is all that is needed to determine the next state. Let $\left\{X_{n}, n=0,1, \ldots\right\}$ be a sequence of random variables with state space $S$. A discretetime Markov chain is defined as

$$
\operatorname{Pr}\left\{X_{n+1}=i_{n+1} \mid X_{0}=i_{0, \ldots}, X_{n}=i_{n}\right\}=\operatorname{Pr}\left\{X_{n+1}=i_{n+1} \mid X_{n}=i_{n}\right\} .
$$

At each step, the system may change its state from state $i \in S$ to state $j \in S$ according to a probability distribution. The changes of state are called transitions, and the probabilities associated with various state changes are called transition probabilities. If state space $S$ is a finite set, the transition probability distribution can be represented by a transition matrix $\mathbf{P}$, with its $(i, j)$ th entry equal to

$$
p_{i j}=\operatorname{Pr}\left\{X_{n+1}=j \mid X_{n}=i\right\}, i, j \in S \text {. }
$$

Since $p_{i j} \in[0,1]$ and $\sum_{j \in S} p_{i j}=1$ for all $i \in S, \mathbf{P}$ is a stochastic square matrix. If the transition matrix $\mathbf{P}$ is the same after each step, then the Markov chain is called time-homogeneous. For such a Markov chain, the $t$-step transition probability can be computed as the $t^{\prime}$ th power of $\mathbf{P}$ denoted as $\mathbf{P}^{t}$. Given an initial distribution $P^{(0)}$, 
the distribution of the chain after $t$ steps can be obtained by $P^{(t)}=P^{(0)} \mathbf{P}^{t}$. Hence, a homogeneous finite Markov chain $\left\{X_{n}, n=0,1, \ldots\right\}$ is completely determined by the initial state $X_{0}$ and transition matrix $\mathbf{P}$.

To understand the long-term behavior (or the limit behavior) of the Markov chain $\left\{X_{n}\right\}$, we first give some (standard) definitions as follows.

DEFINITION 1: A nonnegative square matrix $A$ is said to be regular if there exists $k$ such that for all $i$ and $j$, the $(i, j)$ th entry of $A^{k}$ is positive. (This simply means that for any pair of states some sequence of transitions exists between them.)

DEFINITION 2: A probability distribution $\pi=\left(\pi_{1}, \pi_{2}, \ldots\right)$ is said to be stationary for a transition matrix if it does not change after a round of transitions, that is, with $\boldsymbol{P}=\left(p_{i j}\right)$ then $\pi_{j}=\sum_{i \in S} \pi_{i} p_{i j}, \forall j \in S$.

Definition 3: A set of states $C$ is said to be closed if and only if $\left.p_{i k}^{(n)}\right|_{i \in C, k \notin C}$ for all $n$ and $\sum_{j \in C} p_{i j}=1$ for every $i \in C$.

Definition 4: A finite Markov chain is said to be irreducible if it does not contain any non-empty closed subset of states other than the entire state space $S$. Chains that are not irreducible are said to be reducible.

DEFINITION 5: A Markov chain is said to be aperiodic if and only if the corresponding transition matrix $\boldsymbol{P}$ is regular.

DEFINITION 6: A state $i$ has period $k$ if any return to state $i$ must occur in multiples of $k$ time steps. If $k=1$, then the state is said to be aperiodic.

The following well-known result sets up the foundation of our convergence analysis for the ESWO-II algorithm.

THEOREM 1: (See, e.g., Koralov and Sinai, 2007.) Given a Markov chain with an ergodic matrix of transition probabilities $\boldsymbol{P}$, there exists a unique stationary probability distribution $\pi=\pi_{i}$. The n-step transition probabilities converge to the distribution $\pi$, that is $\lim _{n \rightarrow \infty} p_{i j}^{(n)}=\pi_{j}$. The stationary distribution satisfies $\pi_{j}>0$ for all $j \in S$.

REMARK: The above results can be extended to reducible Markov chain with a single closed set. Note that the meaning of an ergodic matrix is identical to the regular matrix defined in Definition 1.

\section{A Formal Framework for the ESWO-II Algorithm}

Following the earlier general introduction of the ESWO-II, this section gives a formal definition and framework for ESWO-II. It is based on a Markov chain and the state transition point of view, and recall that the main difference between ESWO-II and ESWO is that it will make simple probabilistic choices, rather than heuristic ones. 


\subsection{State Transitions by the ESWO-II Algorithm}

Five operations are performed in sequence at each iteration of the ESWO-II algorithm. They are analysis, selection, mutation, prioritization and construction. The first analysis operation does not alter the current state on its own, as it simply serves as an agent to collect inherent information, in particular the local fitness values of individual components, of the current state.

The second operator, selection, uses the result of the preceding analysis operator and changes the present state by removing the set of selected components from the current solution. However, it does not generate a destination state which is defined as a complete assignment of all components. Hence, we refer to the state after the selection as an intermediate state. Whenever no component has been selected, the intermediate state remains the current state. However, if one or more components have been selected, the intermediate state is an incomplete assignment together with a set of unassigned components. As the selected components are removed, the exact information about the source assignment is thus lost. All possible destination states with the same complete assignments, including the original source, are treated uniformly as seen from the intermediate state. Let $x$ be a source state, and $x^{\prime}$ be an intermediate state. For the selection operator, we write its transition probability from $x$ to $x^{\prime}$ as $p_{S}\left(X_{n+1}=x^{\prime} \mid X_{n}=x\right)$, or $p_{S}\left(x^{\prime} \mid x\right)$ for short.

The third operator, mutation, changes the present intermediate state by further removing the set of mutated components from the current (partial) solution. Like the selection operator, it does not generate a destination state. Hence, the state after mutation remains an intermediate state. Likewise, all destination states with the same complete assignments are treated uniformly as seen from the current intermediate state. Let $x^{\prime}$ be a previous intermediate state, and $x^{\prime \prime}$ be a present intermediate state. For the mutation operator, we write its transition probability from $x^{\prime}$ to $x^{\prime \prime}$ as $p_{\mathrm{M}}\left(X_{n+1}=x^{\prime \prime} \mid X_{n}=x^{\prime}\right)$, or $p_{\mathrm{M}}\left(x^{\prime \prime} \mid x^{\prime}\right)$ for short.

The fourth operation, prioritization, would not directly alter the present intermediate state on its own, but simply use the information of the analysis operator to create the priority order in which the construction operator builds new solutions. However, it is only used in ESWO and not ESWO-II and is only included here for completeness.

The fifth operator, construction, in ESWO-II finally generates a new destination state from the present intermediate state by carrying out probabilistic choices among different possible destination states. In comparison, in ESWO, for construction, the priority order would be used along with partially deterministic algorithms, e.g., greedy heuristics that break ties randomly. Let $x^{\prime \prime}$ be an intermediate state, and $x^{\prime \prime \prime}$ be a destination state. For the construction operator, we write its transition probability from $x^{\prime \prime}$ to $x^{\prime \prime \prime}$ as $p_{C}\left(X_{n+1}=x^{\prime \prime \prime} \mid X_{n}=x^{\prime \prime}\right)$, or $p_{C}\left(x^{\prime \prime \prime} \mid x^{\prime \prime}\right)$ for short.

Assume a solution $x^{(j)} \in S$ consists of $m$ componential variables $x_{i}$, denoted as $x^{(j)}=\left(x_{1}, \ldots, x_{m}\right)^{(j)}$, and each $x_{i}$ may take one of the $n$ values, that is, $x_{i} \in\left\{j_{1}^{(i)}, \ldots, j_{n}^{(i)}\right\}$. The $n$ values for each variable are not essential but simplify the equations. A variable instantiation, that is, the assignment of a value $j_{k}^{(i)}$ to a variable, is denoted by $x_{i}=j_{k}^{(i)}$. A solution $s \in S$ is a complete assignment in which each variable has a domain value assigned, regardless of the satisfiability on constraint set $\Omega$.

The selection operator can be regarded as a linear map from space $S$ of cardinality $n^{m}$ to space $S^{\#}$ of cardinality $(n+1)^{m}$, as each variable $x_{i}$ after selection can take an additional value denoted as \#. A \# symbol means that the variable will be instantiated 


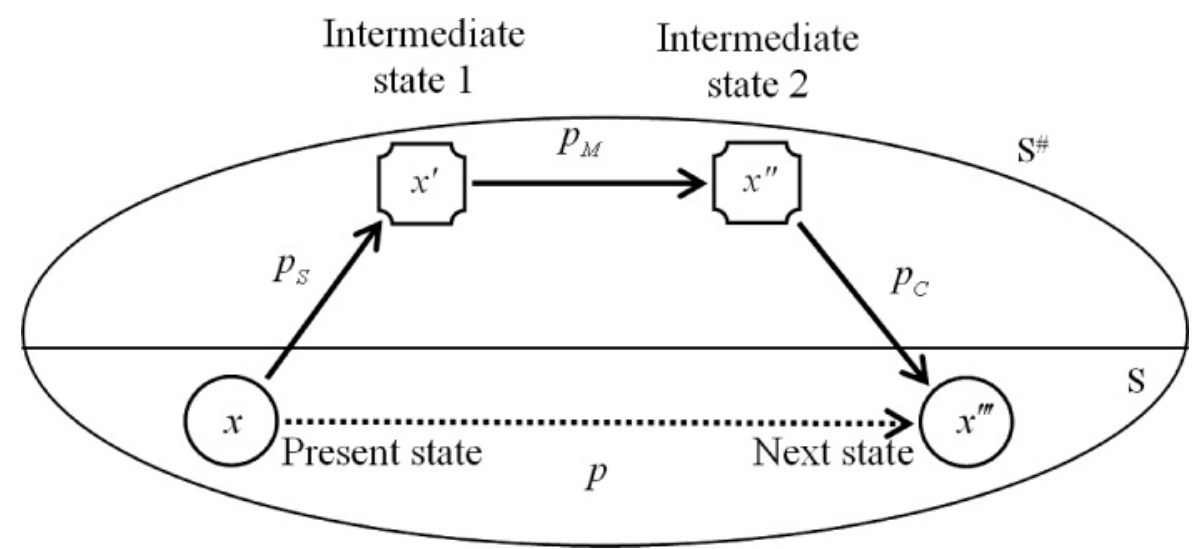

Figure 1: State transition graph of the ESWO-II algorithm.

later when it is marked as selected. That is, a mix of \# and non-\# symbols is a partial assignment rather than a complete assignment.

At each iteration of the ESWO-II algorithm, a state transition is carried out from a source state to a destination state (as shown in Figure 1). This corresponds to a one-step move in the state graph. We can see that, given a present state, the probability of being in a future state is conditionally independent of the past states. Hence, each step of transitions between complete states has Markov properties.

After defining the state transitions of selection, mutation, and construction, we are able to give an expression for the overall transition probability between any two connected states. We formulate the transition probability from the source state $x$ to the destination state $x^{\prime \prime \prime}$, via two intermediate states of $x^{\prime}$ and $x^{\prime \prime}$, as

$$
p\left(x^{\prime \prime \prime} \mid x\right)=p_{\mathrm{S}}\left(x^{\prime} \mid x\right) \cdot p_{\mathrm{M}}\left(x^{\prime \prime} \mid x^{\prime}\right) \cdot p_{\mathrm{C}}\left(x^{\prime \prime \prime} \mid x^{\prime \prime}\right) .
$$

Equation (3) forms the basis for our following Markov chain analysis. We aim to derive some global convergence properties and trace a stationary probability distribution, for an insight into the working of the ESWO-II algorithm.

\subsection{The Running Example}

Throughout this paper, in order to explicitly illustrate the formalism and also to provide empirical results, we use a single running example using just three $0 / 1$ variables $x_{i}$. The search space $S$ has $2^{3}=8$ states, and the intermediate space $S^{\#}$ has $3^{3}=27$ states. We use a superscript in parentheses to index the search space $S$, and will use the fixed numeric order $[000,001,010,011,100,101,110,111]$ so that in this case $j$ is actually just one plus the numeric value of the string when regarded as a binary number. Hence, in this case, a solution $x^{(j)}$ with $j \in[1, \ldots, 8]$ consists of three components denoted as $x^{(j)}=\left(x_{1}, x_{2}, x_{3}\right)^{(j)}$ and each component can only take two values of either 1 or 0 , for example, $x^{(5)}=(1,0,0)$.

We will also take the fitness to be

$$
f\left(x^{(j)}\right)=4 x_{1}+2 x_{2}+x_{3}+1=j,
$$


that is, one plus the value of $x$ when regarded as a binary number. This is a very simple function, but still allows interesting observations to be made.

\subsection{The Analysis Operator}

The analysis operator does not affect the state but instead produces information about the correctness of each element $x_{i}$ of the current solution.

Specifically, it produces a number $g\left(x_{i} \mid x^{(j)}\right) \in[0,1]$ that is the local fitness value of $x_{i}$ in solution $x^{(j)}$. Larger values of $g\left(x_{i} \mid x^{(j)}\right)$ are taken to correspond to values that are considered to be better. However, the fitness is generally only a local and heuristic estimate and thus cannot be considered as a certain indicator of which components really are performing less well than they could.

In a specific algorithm, these estimates are likely to be made heuristically, and thus are difficult to model in general, so in the running example we will make the following simple modeling choice. It is clear that it is always better to have $x_{i}=1$ than $x_{i}=0$. However, we do not want the analysis to have perfect information. Instead, we also want to model that the local fitness could make a mistake and not lead to the global optimum. Hence, we will take it that the analysis gives

$$
g\left(x_{i} \mid x^{(j)}\right)=\left\{\begin{array}{ll}
1 / 3, & \text { if } x_{i}=0 ; \\
2 / 3, & \text { if } x_{i}=1 ;
\end{array} \forall i \in\{1,2,3\}, j \in\{1, \ldots, 8\}\right.
$$

\subsection{The Selection Operator}

Using the local fitness values, $g\left(x_{i} \mid x^{(j)}\right)$, calculated by the preceding analysis operator, the probability that component $x_{i}$ is selected from $x^{(j)}$ to convert to a \# is taken to be

$$
s_{i}^{(j)}=\operatorname{Pr}\left\{x_{i} \text { is selected from } x^{(j)}\right\}=\frac{1-g\left(x_{i} \mid x^{(j)}\right)}{S_{\text {norm }}^{(j)}}, \quad \forall i \in\{1, \ldots, m\}, x^{(j)} \in S
$$

where $S_{\text {norm }}^{(j)}$ is a normalization factor that is used to control the average number of elements that are selected from state $j$.

Let $p_{\mathrm{S}}\left(X_{n+1}=x^{\prime} \mid X_{n}=x\right)$ be the conditional probability that intermediate state $x^{\prime}=$ $\left(x_{1}^{\prime}, \ldots, x_{m}^{\prime}\right)$ is generated from present state $x=\left(x_{1}, \ldots, x_{m}\right)$ by the selection operator, then:

$$
p_{S}\left(X_{n+1}=x^{\prime} \mid X_{n}=x\right)=\left\{\begin{array}{l}
0, \quad \text { if } \quad \exists i \in\{1, \ldots, m\}, \quad x_{i} \neq x_{i}^{\prime} \text { and } x_{i}^{\prime} \neq \# \\
\prod_{i=1}^{m} s_{i}^{(j)}\left(1-s_{i}^{(j)}\right)^{1-D\left(x_{i}, x_{i}^{\prime}\right)}, \quad \text { otherwise }
\end{array}\right.
$$

where

$$
D\left(x_{i}, x_{i}^{\prime}\right)= \begin{cases}1, & \text { if } x_{i} \neq \# \text { and } x_{i}^{\prime}=\# \\ 0, & \text { if } x_{i}=x_{i}^{\prime} \neq \#\end{cases}
$$

Of course, in a practical ESWO algorithm this selection might be done using heuristics, but the choice here allows analytical modeling.

EXAMPLE 1: State transitions by a simple selection operator. 
Table 1: The transition probabilities for the selection operator.

\begin{tabular}{cccccccccccc}
\hline$x^{(j)}$ & $s_{1}^{(j)}$ & $s_{2}^{(j)}$ & $s_{3}^{(j)}$ & $s_{j 1}$ & $s_{j 2}$ & $s_{j 3}$ & $s_{j 4}$ & $s_{j 5}$ & $s_{j 6}$ & $s_{j 7}$ & $s_{j 8}$ \\
\hline 000 & $1 / 3$ & $1 / 3$ & $1 / 3$ & $8 / 27$ & $4 / 27$ & $4 / 27$ & $4 / 27$ & $2 / 27$ & $2 / 27$ & $2 / 27$ & $1 / 27$ \\
001 & $2 / 5$ & $2 / 5$ & $1 / 5$ & $36 / 125$ & $9 / 125$ & $24 / 125$ & $24 / 125$ & $16 / 125$ & $6 / 125$ & $6 / 125$ & $4 / 125$ \\
010 & $2 / 5$ & $1 / 5$ & $2 / 5$ & $36 / 125$ & $24 / 125$ & $9 / 125$ & $24 / 125$ & $6 / 125$ & $16 / 125$ & $6 / 125$ & $4 / 125$ \\
011 & $1 / 2$ & $1 / 4$ & $1 / 4$ & $9 / 32$ & $3 / 32$ & $3 / 32$ & $9 / 32$ & $3 / 32$ & $3 / 32$ & $1 / 32$ & $1 / 32$ \\
100 & $1 / 5$ & $2 / 5$ & $2 / 5$ & $36 / 125$ & $24 / 125$ & $24 / 125$ & $9 / 125$ & $6 / 125$ & $6 / 125$ & $16 / 125$ & $4 / 125$ \\
101 & $1 / 4$ & $1 / 2$ & $1 / 4$ & $9 / 32$ & $3 / 32$ & $9 / 32$ & $3 / 32$ & $3 / 32$ & $1 / 32$ & $3 / 32$ & $1 / 32$ \\
110 & $1 / 4$ & $1 / 4$ & $1 / 2$ & $9 / 32$ & $9 / 32$ & $3 / 32$ & $3 / 32$ & $1 / 32$ & $3 / 32$ & $3 / 32$ & $1 / 32$ \\
111 & $1 / 3$ & $1 / 3$ & $1 / 3$ & $8 / 27$ & $4 / 27$ & $4 / 27$ & $4 / 27$ & $2 / 27$ & $2 / 27$ & $2 / 27$ & $1 / 27$ \\
\hline
\end{tabular}

We use the local fitness from Equation (5). Also, in this paper, we will take

$$
S_{\text {norm }}^{(j)}=\sum_{k=1}^{m}\left(1-g\left(x_{k} \mid x^{(j)}\right)\right), \quad \forall i \in\{1, \ldots, m\}, \quad x^{(j)} \in S
$$

and thus $\sum_{i=1}^{3} s_{i}^{(j)}=1$ for all $j$. Hence, in our simple running example, independently of the state $j$, on average, a single bit will be selected for conversion to a \#. One can say that together the analysis and selection believe a 0 is incorrect and so will often convert one of the 0 s to a \# but they cannot be certain and so will also sometimes select a 1 for conversion to \#.

Computation of the transition matrix is straightforward; for example, $S_{\text {norm }}^{000}=3 *$ $(1-1 / 3)=2$, giving $s_{0}^{000}=(1-1 / 3) / 2=1 / 3$. Since the bits are selected independently, for any solution $x^{(j)}$, the various transition probabilities are simply

$$
\begin{aligned}
& s_{j 1} \equiv p_{\mathrm{S}}\left\{X_{n+1}=\left(x_{1}, x_{2}, x_{3}\right)^{(j)} \mid X_{n}=\left(x_{1}, x_{2}, x_{3}\right)^{(j)}\right\}=\left(1-s_{1}^{(j)}\right)\left(1-s_{2}^{(j)}\right)\left(1-s_{3}^{(j)}\right), \\
& s_{j 2} \equiv p_{\mathrm{S}}\left\{X_{n+1}=\left(x_{1}, x_{2}, \#\right)^{(j)} \mid X_{n}=\left(x_{1}, x_{2}, x_{3}\right)^{(j)}\right\}=\left(1-s_{1}^{(j)}\right)\left(1-s_{2}^{(j)}\right) s_{3}^{(j)}, \\
& s_{j 3} \equiv p_{\mathrm{S}}\left\{X_{n+1}=\left(x_{1}, \#, x_{3}\right)^{(j)} \mid X_{n}=\left(x_{1}, x_{2}, x_{3}\right)^{(j)}\right\}=\left(1-s_{1}^{(j)}\right) s_{2}^{(j)}\left(1-s_{3}^{(j)}\right), \\
& s_{j 4} \equiv p_{\mathrm{S}}\left\{X_{n+1}=\left(\#, x_{2}, x_{3}\right)^{(j)} \mid X_{n}=\left(x_{1}, x_{2}, x_{3}\right)^{(j)}\right\}=s_{1}^{(j)}\left(1-s_{2}^{(j)}\right)\left(1-s_{3}^{(j)}\right), \\
& s_{j 5} \equiv p_{\mathrm{S}}\left\{X_{n+1}=\left(\#, \#, x_{3}\right)^{(j)} \mid X_{n}=\left(x_{1}, x_{2}, x_{3}\right)^{(j)}\right\}=s_{1}^{(j)} s_{2}^{(j)}\left(1-s_{3}^{(j)}\right), \\
& s_{j 6} \equiv p_{\mathrm{S}}\left\{X_{n+1}=\left(\#, x_{2}, \#\right)^{(j)} \mid X_{n}=\left(x_{1}, x_{2}, x_{3}\right)^{(j)}\right\}=s_{1}^{(j)}\left(1-s_{2}^{(j)}\right) s_{3}^{(j)}, \\
& s_{j 7} \equiv p_{\mathrm{S}}\left\{X_{n+1}=\left(x_{1}, \#, \#\right)^{(j)} \mid X_{n}=\left(x_{1}, x_{2}, x_{3}\right)^{(j)}\right\}=\left(1-s_{1}^{(j)}\right) s_{2}^{(j)} s_{3}^{(j)}, \\
& s_{j 8} \equiv p_{\mathrm{S}}\left\{X_{n+1}=(\#, \#, \#)^{(j)} \mid X_{n}=\left(x_{1}, x_{2}, x_{3}\right)^{(j)}\right\}=s_{1}^{(j)} s_{2}^{(j)} s_{3}^{(j)} .
\end{aligned}
$$

Table 1 shows the detailed values of all non-zero transition probabilities for the selection operator. Note that, except for the symmetric states 000 and 111, a 0 bit is precisely twice as likely to be selected as a 1 . Table 2 shows the structure of its associated transition matrix $\mathbf{S}_{8 \times 27}$.

It is also important for understanding the later results to remark that the selector does not know the details of the full global fitness function. It knows that a 1 is better than a 0 and so might be thought of as trying to maximize the number of $1 \mathrm{~s}$; however, it does not know that 011 is less fit than 100 despite having more $1 \mathrm{~s}$. 


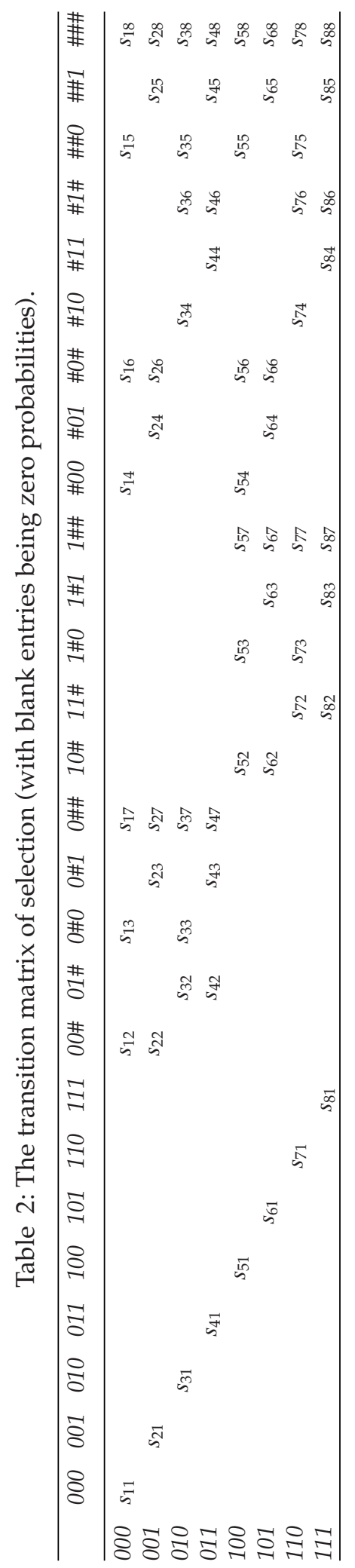




\subsection{The Mutation Operator}

The mutation operator is a map from space $S^{\#}$ to itself that, independently for each part of the solution, will randomly convert a non-\# to a \# (for reinstantiation by the later construction operator). It is similar to the selection operator, except that the mutations do not use any analysis of the solution but are purely random, and also the formalism has to allow the possibility that the element is already a \# due to the preceding selection step.

The probabilities that any non-\# component $x_{i}^{\prime}$ from tuple $x^{\prime}=\left(x_{1}^{\prime}, \ldots, x_{m}^{\prime}\right)$ is converted to a \# is taken to be a mutation probability $q_{\mathrm{M}} \in[0,1]$. Note that, for simplicity, we take the mutation probability to be the same for all components, $i$, but this could easily be extended if desired. It is usually taken to be a non-zero so as to increase diversification, and to be a constant; though it could, if desired, be allowed to change on each iteration.

Let $p_{\mathrm{M}}\left(X_{n+1}=x^{\prime \prime} \mid X_{n}=x^{\prime}\right)$ be the conditional probability that tuple $x^{\prime \prime}=\left(x_{1}^{\prime \prime}, \ldots, x_{m}^{\prime \prime}\right)$ is generated from tuple $x^{\prime}=\left(x_{1}^{\prime}, \ldots, x_{m}^{\prime}\right)$ by the mutation operator, then:

$$
p_{\mathrm{M}}\left(X_{n+1}=x^{\prime \prime} \mid X_{n}=x^{\prime}\right)=\left\{\begin{array}{l}
0, \quad \text { iff } \quad \exists i \in\{1, \ldots, m\}, x_{i}^{\prime} \neq x_{i}^{\prime \prime} \text { and } x_{i}^{\prime \prime} \neq \# ; \\
\prod_{i=1}^{m}\left(q_{\mathrm{M}}^{D\left(x_{i}^{\prime}, x_{i}^{\prime \prime}\right)}\left(1-q_{\mathrm{M}}\right)^{1-D\left(x_{i}^{\prime}, x_{i}^{\prime \prime}\right)}\right)^{1-\lambda_{i}}, \text { otherwise }
\end{array}\right.
$$

where the constants

$$
\begin{aligned}
D\left(x_{i}^{\prime}, x_{i}^{\prime \prime}\right) & = \begin{cases}1, & \text { if } x_{i}^{\prime} \neq \# \text { and } x_{i}^{\prime \prime}=\# ; \\
0, & \text { if } x_{i}^{\prime} \neq \# \text { and } x_{i}^{\prime \prime} \neq \#,\end{cases} \\
\lambda_{i} & = \begin{cases}1, & \text { if } x_{i}^{\prime}=x_{i}^{\prime \prime}=\# ; \\
0, & \text { otherwise }\end{cases}
\end{aligned}
$$

simply capture that the mutation is only permitted to change a non-\# to a \#.

EXAMPLE 2: State transitions by a mutation operator.

Returning to the running example, and taking $q_{\mathrm{M}}=1 / 10$, the resulting transition matrix $\mathbf{M}_{27 \times 27}$ for the mutation step, is given in explicit detail in Table 3 .

\subsection{The Construction Operator}

The last operator, construction, is a linear map from space $S^{\#}$ back to the original space $S$. Let $p_{C}\left(X_{n+1}=x^{\prime \prime \prime} \mid X_{n}=x^{\prime \prime}\right)$ be the conditional probability that tuple $x^{\prime \prime \prime}=\left(x_{1}^{\prime \prime \prime}, \ldots, x_{m}^{\prime \prime \prime}\right)$ is generated from tuple $x^{\prime \prime}=\left(x_{1}^{\prime \prime}, \ldots, x_{m}^{\prime \prime}\right)$ by the construction operator. Let $N\left(x^{\prime \prime}\right)$ be the set of all possible destination states that can be constructed from intermediate state $x^{\prime \prime}$. Clearly, $\left|N\left(x^{\prime \prime}\right)\right|=n^{l_{\#}}$, where $l_{\#}$ is the number of \# in $x^{\prime \prime}$.

Suppose that $f(\cdot)$ is the global fitness function and that, without loss of generality, we have a maximization problem for destination states in space $S$, and then the intent of the constructor is that it should be biased toward states with larger values of $f$. However, since a locally optimal solution within $N\left(x^{\prime \prime}\right)$ could still be misleading for obtaining global solutions, we still want the constructor to be probabilistic and so allow 


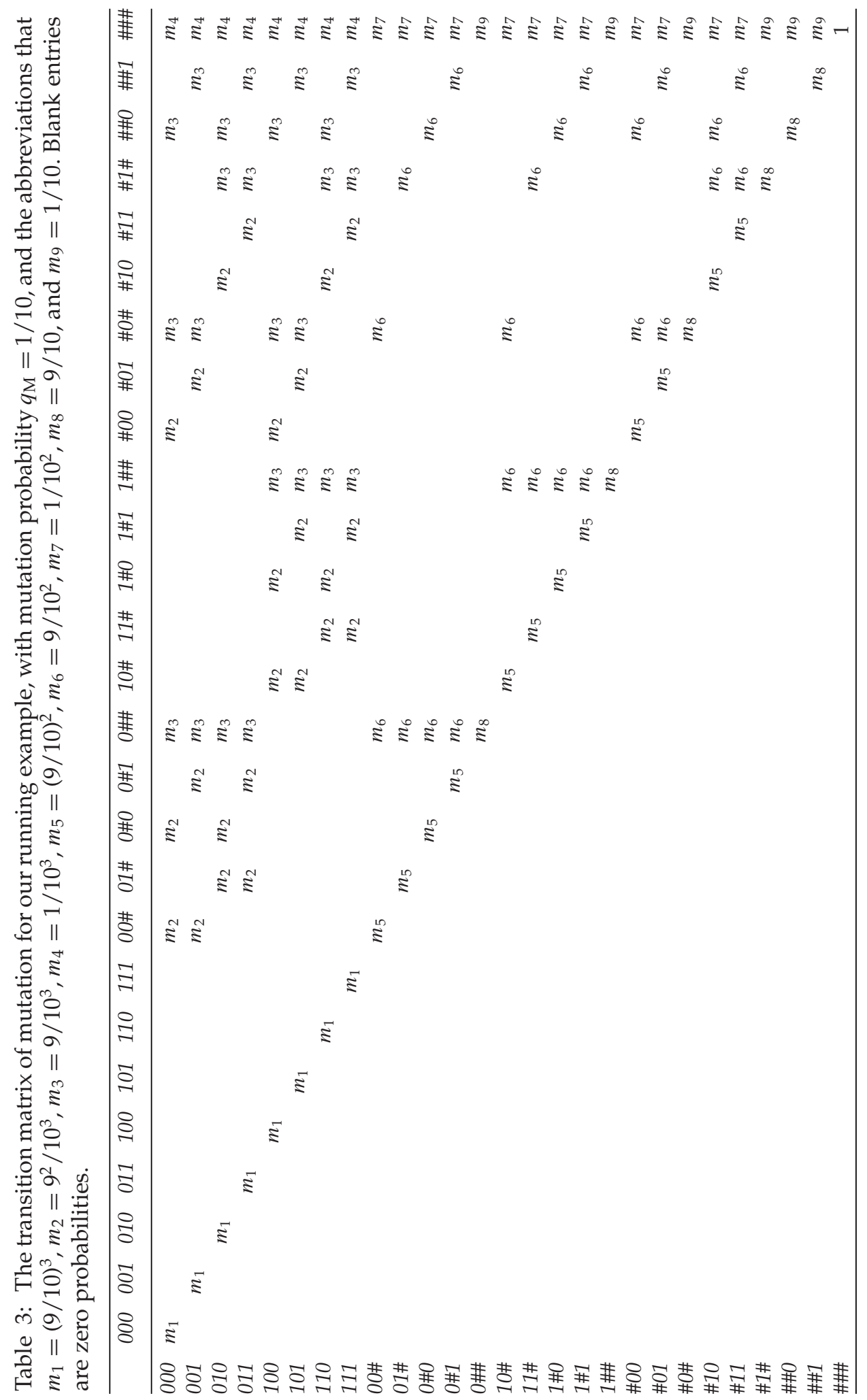


diversification in the search. There are many possibilities for how to do this probabilistic bias, but here we initially take:

$$
p_{C}\left(X_{n+1}=x^{\prime \prime \prime} \mid X_{n}=x^{\prime \prime}\right)=\left\{\begin{array}{l}
0, \text { if } \exists i \in\{1, \ldots, m\}, \quad x_{i}^{\prime \prime} \neq \# \text { and } x_{i}^{\prime \prime \prime} \neq x_{i}^{\prime \prime} ; \\
1, \text { if } \forall i \in\{1, \ldots, m\}, x_{i}^{\prime \prime}=x_{i}^{\prime \prime \prime} \\
\frac{f\left(x^{\prime \prime \prime}\right)}{\sum_{X \in N\left(x^{\prime \prime}\right)} f(X)}, \text { otherwise. }
\end{array}\right.
$$

The first two cases of Equation (13) are automatic constraints in that they merely say that the constructor can only assign values to unassigned, \#, variables. We will assume that $f(x)>0, \forall x \in S$, which, if needed, is easily achieved by adding a constant to $f$. This is also the reason for the +1 in Equation (4).

A method to implement this would be to enumerate the elements of $\left|N\left(x^{\prime \prime}\right)\right|$ and then perform a tournament style selection weighted by fitness. However, $\left|N\left(x^{\prime \prime}\right)\right|=n^{l_{\#}}$, and so increases exponentially with $l_{\#}$, and would quickly become impractical for larger values of $l_{\# \text {. }}$.

Hence, to reduce the amount of computation required during the construction step, we introduce a threshold parameter $t$, and if the number of \#s exceeds $t$, a simple unbiased random construction is performed in which all the neighboring states have an equal probability to be reached. This gives a revised version of Equation (13), and the constructor that we use is defined by

$$
p_{C}\left(X_{n+1}=x^{\prime \prime \prime} \mid X_{n}=x^{\prime \prime}\right)=\left\{\begin{array}{l}
0, \text { if } \exists i \in\{1, \ldots, m\}, \quad x_{i}^{\prime \prime} \neq \# \text { and } x_{i}^{\prime \prime \prime} \neq x_{i}^{\prime \prime} ; \\
1, \text { if } \forall i \in\{1, \ldots, m\}, \quad x_{i}^{\prime \prime}=x_{i}^{\prime \prime \prime} \\
1 / n^{l \#}, \text { if } l_{\#}>t ; \\
\frac{f\left(x^{\prime \prime \prime}\right)}{\sum_{X \in N\left(x^{\prime \prime}\right)} f(X)}, \text { otherwise. }
\end{array}\right.
$$

Note that $t=0$ corresponds to a random constructor, and $t=m$ to Equation (13). We will use C_L $t$ to refer to different choices for $t$.

Equation (14) is a specific choice for a constructor and the primary choice that defines the algorithm to be ESWO-II rather than a general ESWO. Recall that the intent of the randomized constructive method is to have some variability but still to favor the construction of fitter solutions. However, in order to be able to perform the analysis, we still wanted something with a declarative definition, rather than only being defined using some heuristic construction method.

Note that the constructor has no memory of the state of the prior state of a \# before it was unassigned. The selection or mutation that converts an element to a \# does not necessarily ultimately force it to change. This makes it different from standard local search in which a move is intrinsically to a different state; the possibility to keep the same state presumably plays a similar role as the rejection of moves under a metaheuristic such as simulated annealing.

EXAMPLE 3: State transitions by a simple construction operator.

In addition to the above example problem, when we use Equation (14) and the number of \#s at a present intermediate state is one, its possible destination states are probabilistically chosen according to their global fitness values. When the number of \#s is larger than one, each possible destination state will be reached with the same 
Table 4: The transition matrix of construction (with blank entries being zero probabilities).

\begin{tabular}{|c|c|c|c|c|c|c|c|c|}
\hline & 000 & 001 & 010 & 011 & 100 & 101 & 110 & 111 \\
\hline 000 & 1 & & & & & & & \\
\hline 001 & & 1 & & & & & & \\
\hline 010 & & & 1 & & & & & \\
\hline 011 & & & & 1 & & & & \\
\hline 100 & & & & & 1 & & & \\
\hline 101 & & & & & & 1 & & \\
\hline 110 & & & & & & & 1 & \\
\hline 111 & & & & & & & & 1 \\
\hline 00\# & $1 /(1+2)$ & $2 /(1+2)$ & & & & & & \\
\hline 01\# & & & $3 /(3+4)$ & $4 /(3+4)$ & & & & \\
\hline $0 \# 0$ & $1 /(1+3)$ & & $3 /(1+3)$ & & & & & \\
\hline $0 \# 1$ & & $2 /(2+4)$ & & $4 /(2+4)$ & & & & \\
\hline $0 \# \#$ & $1 / 4$ & $1 / 4$ & $1 / 4$ & $1 / 4$ & & & & \\
\hline $10 \#$ & & & & & $5 /(5+6)$ & $6 /(5+6)$ & & \\
\hline 11\# & & & & & & & $7 /(7+8)$ & $8 /(7+8)$ \\
\hline 1\#0 & & & & & $5 /(5+7)$ & & $7 /(5+7)$ & \\
\hline 1\#1 & & & & & & $6 /(6+8)$ & & $8 /(6+8)$ \\
\hline 1\#\# & & & & & $1 / 4$ & $1 / 4$ & $1 / 4$ & $1 / 4$ \\
\hline$\# 00$ & $1 /(1+5)$ & & & & $5 /(1+5)$ & & & \\
\hline \#01 & & $2 /(2+6)$ & & & & $6 /(2+6)$ & & \\
\hline \#0\# & $1 / 4$ & $1 / 4$ & & & $1 / 4$ & $1 / 4$ & & \\
\hline$\# 10$ & & & $3 /(3+7)$ & & & & $7 /(3+7)$ & \\
\hline \#11 & & & & $4 /(4+8)$ & & & & $8 /(4+8)$ \\
\hline \#1\# & & & $1 / 4$ & $1 / 4$ & & & $1 / 4$ & $1 / 4$ \\
\hline \#\#0 & $1 / 4$ & & $1 / 4$ & & $1 / 4$ & & $1 / 4$ & \\
\hline \#\#1 & & $1 / 4$ & & $1 / 4$ & & $1 / 4$ & & $1 / 4$ \\
\hline \#\#\# & $1 / 8$ & $1 / 8$ & $1 / 8$ & $1 / 8$ & $1 / 8$ & $1 / 8$ & $1 / 8$ & $1 / 8$ \\
\hline
\end{tabular}

probability, regardless of its global fitness value. Recall from Equation (4) that the global fitness function is simply one plus the decimal value of a binary vector when it is regarded as a binary number.

Table 4 shows the probability values of transition matrix $\mathbf{C}_{27 \times 8}$ after the construction step.

\section{Markov Chain Model for ESWO-II}

We first give a basic definition that is a natural extension to the standard stochastic matrix. It arises because of the way that the search moves through intermediate states and spaces of different dimensions.

Definition 7: A matrix $\mathbf{T}=\left[t_{i j}\right]_{m \times n}$ is row-stochastic if $\mathbf{T}$ is not necessarily a square matrix (i.e., $m$ does not necessarily equal $n$ ), but still satisfies: $t_{i j} \in[0,1]$ and $\sum_{j=1}^{n} t_{i j}=1$ for all $i=\{1, \ldots, m\}$.

The above definition just says that $\mathrm{T}$ defines a probabilistic transition in which no "mass" is lost. We can easily verify the following result. 
LEMMA 1: Let $S$ be an $m \times n$ row-stochastic matrix, $M$ an $n \times n$ stochastic matrix, and $C$ an $n \times m$ row-stochastic matrix. Then the product S.M.C is a stochastic matrix.

Assume that a solution consists of $m$ variables $x_{i}, i \in\{1, \ldots, m\}$, and each $x_{i}$ may take one of the $n$ values, that is, $x_{i} \in\left\{j_{1}, \ldots, j_{n}\right\}$. The selection operator carries out a linear map from space $S$ of cardinality $n^{m}$ to space $S^{\prime}$ of cardinality $(n+1)^{m}$, with a transition matrix $\mathbf{S}=\left[s_{i j}\right]_{n^{m} \times(n+1)^{m}}$ defined by Equations (6), (7), and (8). Next, the mutation operator carries out a linear map from space $S^{\prime}$ of cardinality $(n+1)^{m}$ to itself, with transition matrix $\mathbf{M}=\left[m_{i j}\right]_{(n+1)^{m} \times(n+1)^{m}}$ defined by Equations (8), (9), and (10). Finally, the construction operator carries out a linear map from space $S^{\prime}$ of cardinality $(n+1)^{m}$ back to the original space $S$ of cardinality $n^{m}$, with a transition matrix $\mathbf{C}=$ $\left[c_{i j}\right]_{(n+1)^{m} \times n^{m}}$ defined by Equation (13) or Equation (14). By Lemma 1, we have the following statement immediately.

LEMMA 2: All state transitions of the ESWO-II algorithm with component selection, fixed rate mutation and solution construction are probabilistic (in the sense of it being possible to model with a Markov stochastic process).

The three operators of selection, mutation, and construction work as a whole to carry out a linear transformation from state space $S$ to itself, by an overall transition matrix $\mathbf{P}$ which is the product of three intermediate-transformation matrices corresponding to the individual operators. Note that the Markov chain of the ESWO-II algorithm is homogeneous as none of the matrices depend on time. Hence, many standard theorems of Markov analysis apply directly.

In theoretical studies of genetic algorithms, the most well-known class of evolutionary algorithms, an overall transition matrix is also achieved through the multiplication of matrices corresponding to the transition operators of selection, crossover, and mutation (Rudolph, 1994; Agapie, 1998). Although it has the same names for two of the operators, the state transition process of our proposed ESWO-II algorithm is quite different from that of genetic algorithms. Each of the genetic algorithms' operators is a map within the same problem space (i.e., endomorphism), while this is not the case for the ESWO-II algorithm.

THEOREM 2: If the mutation probability is non-zero, $q_{\mathrm{M}}>0$, then the ESWO-II algorithm using the constructor of Equation (13) or (14) visits the global optimal set with probability one.

ProOf (SHORT): As long as the mutation rate is strictly positive, $q_{\mathrm{M}}>0$, then on any iteration there is a strictly positive probability that all elements will be unassigned and converted to a \#. Also, by construction, there is a strictly positive probability of generating any optimal solution. Hence, by going through the state of purely \#s there is a non-zero chance of reaching an optimal state in a single iteration.

Note that the theorem also applies if $q_{\mathrm{M}}=0$ as long as the selection probability is non-zero. The following longer proof sketch is given in fuller detail simply because it is useful for actual calculations for the tables given later.

Proof (Detailed): To simplify the presentation, we define $\alpha=n^{m}$ and $\beta=(n+1)^{m}-$ $n^{m}$ so the pure states are indexed by $1, \ldots, \alpha$ and the states with at least one \# are indexed by $1, \ldots, \beta$. 
The selection transition matrix can be partitioned into the following two block matrices, $\mathbf{S}=\left[\begin{array}{ll}\mathbf{D}_{\alpha} & \mathbf{S}_{\alpha \times \beta}^{\prime}\end{array}\right]$ where diagonal matrix $\mathbf{D}$, with elements $d_{k}$, corresponds to the situations of no components being selected. It is convenient to write $d_{k l}=d_{k}$ if $k=l$ and $d_{k l}=0$ if $k \neq l$. The rectangular matrix $\mathbf{S}^{\prime}$ corresponds to the situations of at least one of the components being selected.

The transition matrix $\mathbf{M}$ of mutation can be partitioned into the following four block matrices $\mathbf{M}=\left[\begin{array}{cc}t \mathbf{I}_{n^{m}} & \mathbf{M}_{\alpha \times \beta}^{\prime} \\ \mathbf{0}_{\beta \times \alpha} & \mathbf{M}_{\beta \times \beta}^{\prime \prime}\end{array}\right]$, where $t=\left(1-q_{\mathbf{M}}\right)^{n^{m}}$ where $q_{\mathrm{M}}$ is the mutation rate. Diagonal matrix $t \mathbf{I}$ and rectangular matrix $\mathbf{M}^{\prime}$ are based on the condition that no component has been previously selected: $t \mathbf{I}$ corresponds to the situation of no components being mutated again, while $\mathbf{M}^{\prime}$ corresponds to the situation of at least one of the components being mutated. Zero matrix $\mathbf{0}$ and upper triangular matrix $\mathbf{M}^{\prime \prime}$ (with all diagonal entries being non-zero) are based on the condition that at least one of the components has been previously selected: 0 means that no destination state is reachable from the intermediate states, and $\mathbf{M}^{\prime \prime}$ corresponds to the situations of components being further mutated from the intermediate states.

The transition matrix $\mathbf{C}$ of construction can be partitioned into the following two block matrices $\mathbf{C}=\left[\begin{array}{c}\mathbf{I}_{\alpha} \\ \mathbf{C}_{\beta \times \alpha}^{\prime}\end{array}\right]$, where identity matrix I means that a state remains the same if no components of it have been selected or mutated, and matrix $\mathbf{C}^{\prime}$ denotes the transition probabilities from intermediate states to destination states by construction.

The overall transition matrix is then

$$
\begin{aligned}
\mathbf{P}=\mathbf{S} \cdot \mathbf{M} \cdot \mathbf{C} & =\left[t \mathbf{D}_{\alpha}+\mathbf{D}_{\alpha} \mathbf{M}_{\alpha \times \beta}^{\prime} \mathbf{C}_{\beta \times \alpha}^{\prime}+\mathbf{S}_{\alpha \times \beta}^{\prime} \mathbf{M}_{\beta \times \beta}^{\prime \prime} \mathbf{C}_{\beta \times \alpha}^{\prime}\right] \\
& =\left[t \mathbf{D}_{\alpha}+\left(\mathbf{D} \cdot \mathbf{M}^{\prime} \cdot \mathbf{C}^{\prime}\right)_{\alpha \times \alpha}+\left(\mathbf{S}^{\prime} \cdot \mathbf{M}^{\prime \prime} \cdot \mathbf{C}^{\prime}\right)_{\alpha \times \alpha}\right] .
\end{aligned}
$$

Writing each term in full then gives

$$
\begin{aligned}
& t \mathbf{D}=\left[\begin{array}{ccc}
t d_{1} & \cdots & 0 \\
\vdots & \ddots & \vdots \\
0 & \cdots & t \\
d_{\alpha}
\end{array}\right] \\
& \mathbf{D} \cdot \mathbf{M}^{\prime} \cdot \mathbf{C}^{\prime}=\left[\begin{array}{ccc}
d_{1} \sum_{i=1}^{\beta} m_{1 i}^{\prime} c_{i 1}^{\prime} & \cdots & d_{1} \sum_{i=1}^{\beta} m_{1 i}^{\prime} c_{i \alpha}^{\prime} \\
\vdots & \ddots & \vdots \\
d_{\alpha} \sum_{i=1}^{\beta} m_{\alpha i}^{\prime} c_{i 1}^{\prime} & \cdots & d_{\alpha} \sum_{i=1}^{\beta} m_{\alpha i}^{\prime} c_{i \alpha}^{\prime}
\end{array}\right] \\
& \mathbf{S}^{\prime} \cdot \mathbf{M}^{\prime \prime} \cdot \mathbf{C}^{\prime}=\left[\begin{array}{ccc}
\sum_{i=1}^{\beta} \sum_{j=1}^{\beta} s_{1 j}^{\prime} m_{j i}^{\prime \prime} c_{i 1}^{\prime} & \cdots & \sum_{i=1}^{\beta} \sum_{j=1}^{\beta} s_{1 j}^{\prime} m_{j i}^{\prime \prime} c_{i \alpha}^{\prime} \\
\vdots & \ddots & \vdots \\
\sum_{i=1}^{\beta} \sum_{j=1}^{\beta} s_{\alpha j}^{\prime} m_{j i}^{\prime \prime} c_{i 1}^{\prime} & \cdots & \sum_{i=1}^{\beta} \sum_{j=1}^{\beta} s_{\alpha j}^{\prime} m_{j i}^{\prime \prime} c_{i \alpha}^{\prime}
\end{array}\right] .
\end{aligned}
$$


Hence, finally, the transition matrix $\mathbf{P}$ is

$$
\left[\begin{array}{ccc}
t d_{1}+d_{1} \sum_{i=1}^{\beta} m_{1 i}^{\prime} c_{i 1}^{\prime}+\sum_{i=1}^{\beta} \sum_{j=1}^{\beta} s_{1 j}^{\prime} m_{j i}^{\prime \prime} c_{i 1}^{\prime} & \cdots & d_{1} \sum_{i=1}^{\beta} m_{1 i}^{\prime} c_{i \alpha}^{\prime}+\sum_{i=1}^{\beta} \sum_{j=1}^{\beta} s_{1 j}^{\prime} m_{j i}^{\prime \prime} c_{i \alpha}^{\prime} \\
\vdots & \ddots & \vdots \\
d_{\alpha} \sum_{i=1}^{\beta} m_{\alpha i}^{\prime} c_{i 1}^{\prime}+\sum_{i=1}^{\beta} \sum_{j=1}^{\beta} s_{\alpha j}^{\prime} m_{j i}^{\prime \prime} c_{i 1}^{\prime} & \cdots & t d_{\alpha}+d_{\alpha} \sum_{i=1}^{\beta} m_{\alpha i}^{\prime} c_{i \alpha}^{\prime}+\sum_{i=1}^{\beta} \sum_{j=1}^{\beta} s_{\alpha j}^{\prime} m_{j i}^{\prime \prime} c_{i \alpha}^{\prime}
\end{array}\right]
$$

or more compactly

$$
P_{k l}=t d_{k l}+d_{k} \sum_{i=1}^{\beta} m_{k i}^{\prime} c_{i l}^{\prime}+\sum_{i=1}^{\beta} \sum_{j=1}^{\beta} s_{k j}^{\prime} m_{j i}^{\prime \prime} c_{i l}^{\prime}
$$

Note that all the terms are nonnegative, hence, for all $k, l$

$$
\begin{aligned}
P_{k l} & \geq d_{k} \sum_{i=1}^{\beta} m_{k i}^{\prime} c_{i l}^{\prime} \\
& \geq d_{k} m_{k \beta}^{\prime} c_{\beta l}^{\prime} \\
& =d_{k} \frac{\left(q_{\mathrm{M}}\right)^{m}}{n^{m}}>0
\end{aligned}
$$

Note that the state indexed by $\beta$ is taken to be the one with purely \#s. This suffices to show that the matrix is regular (and ergodic) for the typical case that $d_{k}>0, q_{\mathrm{M}}>0$ and $c_{\beta l}^{\prime}>0$, meaning that for every element, even if it is not selected, it may be chosen for mutation, and has a probability of reaching any state. Hence, the ESWO-II algorithm visits a global optimum after a finite (possibly very large) number of transition steps, regardless of the starting states, with probability one.

If, furthermore, we assume that the various operators are constants, for example, the mutation probability is constant and non-zero, then it follows by Theorem 1 that there exists a unique stationary probability distribution $\pi=\left(\pi_{1}, \ldots, \pi_{|S|}\right)$, and $\pi_{j}>0$ for $1 \leq j \leq|S|$, and so again the optima have non-zero probability. However, of course, it does not converge in the sense of all of the probability lying only on optimal solutionsbecause all states and not just optima have some non-zero probability.

Naturally, in real world applications, a stochastic algorithm such as ESWO-II always keeps the best solution found over time. Then clearly:

COROLLARY 1: The ESWO-II algorithm, maintaining the best solution found over time, converges to the global optimum.

More formally, under this circumstance, a state becomes a tuple of two solutions $\left(b_{0}, b_{1}\right)$, where $b_{0}$ denotes the previous best solution and $b_{1}$ the current solution (Rudolph, 1994). Obviously, if $b_{0}=s^{*}$ where $s^{*}$ is the only optimal solution of the problem, then all the states (i.e., solution pairs) containing $s^{*}$ constitute a single closed set. 
Hence, by the extension of Theorem 1 to a reducible Markov chain, the probability of remaining in the set of non-closed states converges to zero.

\section{Discussion of Results on Stationary Distribution}

LEMMA 3: Consider a finite Markov chain with a regular transition matrix $\boldsymbol{P}$. If matrix $\boldsymbol{P}$ is symmetric then the stationary probabilities $\left\{\pi_{i}, i \in S\right\}$ are equally distributed, with a value of $1 /|S|$, on state $i$ for all $i \in S$.

Proof: As $\mathbf{P}$ is stochastic, we have $\sum_{i \in S} p_{j i}=1, \forall j \in S$. If $\mathbf{P}$ is symmetric, then $p_{i j}=$ $p_{j i}, \forall i, j \in S \Rightarrow \sum_{i \in S} p_{i j}=1, \forall j \in S \Rightarrow \frac{1}{|S|}=\sum_{i \in S} \frac{1}{|S|} p_{i j}, \forall j \in S$. The vector $\pi^{\prime}$ with a value of $1 /|S|$ on each state is a stationary distribution of $\mathbf{P}$, since the equilibrium equations and the normalizing condition as given in Definition 2 are all satisfied. Furthermore, by Theorem 1 , for any regular transition matrix $\mathbf{P}$, the stationary probability distribution $\pi=\left(\pi_{1}, \ldots, \pi_{|S|}\right)$ is unique. Hence, we can conclude that such a vector $\pi^{\prime}$ is the only stationary distribution if $\mathbf{P}$ is a symmetric regular matrix.

LEMMA 4: For a state of $a \in S$ and an intermediate state $b \in S^{\prime}$, one step of transition by selection and mutation cannot change $a$ to $b$, if and only if one step of transition by construction cannot change $b$ to $a$.

REMARK: This is "by construction" because a selection with mutation can only change a complete assignment to a partial assignment by changing entries to a \# without changing any entries otherwise. The construction can only affect \# entries. In ESWO-II, any such allowed changes also have some probability of happening.

Next, we investigate the conditions on which the stationary probabilities are equally distributed. The following theorem will aid our later discussion.

THEOREM 3: For an ESWO-II algorithm with a randomized selection and a randomized construction, irrespective of the rate of mutation (as long as it is non-zero), the stationary probabilities $\left\{\pi_{i}, i \in S\right\}$ are equally distributed on each state $i$.

By a randomized selector, we just mean one that has no attempt to bias toward any one solution, and so for example $g\left(x_{i_{1}} \mid x^{(j)}\right)=g\left(x_{i_{2}} \mid x^{(j)}\right)$ holds for all $x_{i_{1}}$ and $x_{i_{2}}$; we will denote this by $S_{-}$rand. Similarly, a purely random constructor, C_LO, is, for example, also given by the case $t=0$ of Equation (14), in which all legal transitions are equally likely.

PROOF SKETCH: Since the randomized selection and construction treat all states in $S$ equally, then there is no bias toward any one state and so the only sensible distribution is uniform. A direct proof is possible using Equation (17), but is omitted as it is long and not informative.

EXAMPLE 4: Transition matrices by randomized selection and a randomized construction.

In addition to the previous examples where $f(x)=4 x_{1}+2 x_{2}+x_{3}+1$, we implement various combinations of different types of operators as follows:

- For the selection operator, $\mathbf{S}$ _rand denotes the randomized one as introduced in Theorem 3, $\mathbf{S}$ denotes the one that defines its component fitness $g\left(x_{i} \mid x^{(j)}\right)$ as in 
Table 5: Transition matrices: (a) S_rand $\times$ M_05 $\times$ C_rand, and (b) S_rand $\times$ M_10 $\times$ C_rand.

\begin{tabular}{|c|c|c|c|c|c|c|c|c|c|}
\hline & & 000 & 001 & 010 & 011 & 100 & 101 & 110 & 111 \\
\hline \multirow{10}{*}{ (a) } & 000 & 0.545 & 0.122 & 0.122 & 0.027 & 0.122 & 0.027 & 0.027 & 0.006 \\
\hline & 001 & 0.122 & 0.545 & 0.027 & 0.122 & 0.027 & 0.122 & 0.006 & 0.027 \\
\hline & 010 & 0.122 & 0.027 & 0.545 & 0.122 & 0.027 & 0.006 & 0.122 & 0.027 \\
\hline & 011 & 0.027 & 0.122 & 0.122 & 0.545 & 0.006 & 0.027 & 0.027 & 0.122 \\
\hline & 100 & 0.122 & 0.027 & 0.027 & 0.006 & 0.545 & 0.122 & 0.122 & 0.027 \\
\hline & 101 & 0.027 & 0.122 & 0.006 & 0.027 & 0.122 & 0.545 & 0.027 & 0.122 \\
\hline & 110 & 0.027 & 0.006 & 0.122 & 0.027 & 0.122 & 0.027 & 0.545 & 0.122 \\
\hline & 111 & 0.006 & 0.027 & 0.027 & 0.122 & 0.027 & 0.122 & 0.122 & 0.545 \\
\hline & \multirow[t]{2}{*}{$\pi$} & 0.125 & 0.125 & 0.125 & 0.125 & 0.125 & 0.125 & 0.125 & 0.125 \\
\hline & & 000 & 001 & 010 & 011 & 100 & 101 & 110 & 111 \\
\hline \multirow{9}{*}{ (b) } & 000 & 0.512 & 0.128 & 0.128 & 0.032 & 0.128 & 0.032 & 0.032 & 0.008 \\
\hline & 001 & 0.128 & 0.512 & 0.032 & 0.128 & 0.032 & 0.128 & 0.008 & 0.032 \\
\hline & 010 & 0.128 & 0.032 & 0.512 & 0.128 & 0.032 & 0.008 & 0.128 & 0.032 \\
\hline & 011 & 0.032 & 0.128 & 0.128 & 0.512 & 0.008 & 0.032 & 0.032 & 0.128 \\
\hline & 100 & 0.128 & 0.032 & 0.032 & 0.008 & 0.512 & 0.128 & 0.128 & 0.032 \\
\hline & 101 & 0.032 & 0.128 & 0.008 & 0.032 & 0.128 & 0.512 & 0.032 & 0.128 \\
\hline & 110 & 0.032 & 0.008 & 0.128 & 0.032 & 0.128 & 0.032 & 0.512 & 0.128 \\
\hline & 111 & 0.008 & 0.032 & 0.032 & 0.128 & 0.032 & 0.128 & 0.128 & 0.512 \\
\hline & $\pi$ & 0.125 & 0.125 & 0.125 & 0.125 & 0.125 & 0.125 & 0.125 & 0.125 \\
\hline
\end{tabular}

Equation (5), and S_reve denotes the one that defines its component fitness as $1-g\left(x_{i} \mid x^{(j)}\right)$ (i.e., the reverse of operator $\mathbf{S}$ ).

- For the mutation operator, M_05 denotes the case of $q_{\mathrm{M}}=0.05$ in Equation (10), M_10 the case of $q_{\mathrm{M}}=0.10$, and M_20 the case of $q_{\mathrm{M}}=0.20$.

- For the construction operator, C_rand denotes the randomized one as introduced in Theorem 3, C_L1 denotes the case of $t=1$ in Equation (17), and C_L2 denotes the case of $t=2$.

Table 5 shows two transition matrices of using randomized operators or selection and construction, with different mutation operators (i.e., M_05 and M_10). The computations are standard and are implemented using MATLAB. We can see that the two matrices are different due to different $q_{\mathrm{M}}$ settings, but they are all symmetric matrices and thus have the same equally distributed stationary distribution vectors $\pi$.

As we have seen explicitly, when the steps of selection and construction are all randomized then the process is ergodic and furthermore all states are equally likely. Naturally, the final distribution is not always uniform, and we have the following statement.

COROLLARY 2: For the ESWO-II algorithm, the stationary probability distribution $\left\{\pi_{i}, i \in S\right\}$ on individual states is affected by the strategies of selection, mutation, and construction.

REMARK: Corollary 2 indicates that the stationary probability distribution $\pi=\left(\pi_{i} \mid i \in S\right)$ on individual states is controllable through a proper combination of the selection function, the mutation rate, and the construction function. In particular, without keeping the 
Table 6: Transition matrices: (a) S_reve $\times \mathbf{M} \_10 \times C_{\_} L 1$, and (b) $S \times M_{-} 05 \times C_{-}$L2 .

\begin{tabular}{|c|c|c|c|c|c|c|c|c|c|}
\hline & & 000 & 001 & 010 & 011 & 100 & 101 & 110 & 111 \\
\hline \multirow{10}{*}{ (a) } & 000 & 0.404 & 0.152 & 0.164 & 0.032 & 0.176 & 0.032 & 0.032 & 0.008 \\
\hline & 001 & 0.151 & 0.526 & 0.037 & 0.099 & 0.037 & 0.123 & 0.007 & 0.019 \\
\hline & 010 & 0.130 & 0.037 & 0.544 & 0.106 & 0.037 & 0.007 & 0.118 & 0.019 \\
\hline & 011 & 0.046 & 0.152 & 0.140 & 0.509 & 0.007 & 0.025 & 0.025 & 0.097 \\
\hline & 100 & 0.080 & 0.037 & 0.033 & 0.007 & 0.522 & 0.190 & 0.107 & 0.023 \\
\hline & 101 & 0.046 & 0.108 & 0.007 & 0.025 & 0.144 & 0.557 & 0.025 & 0.089 \\
\hline & 110 & 0.046 & 0.007 & 0.117 & 0.025 & 0.137 & 0.025 & 0.558 & 0.086 \\
\hline & 111 & 0.008 & 0.032 & 0.032 & 0.104 & 0.032 & 0.118 & 0.123 & 0.551 \\
\hline & $\pi$ & 0.113 & 0.133 & 0.139 & 0.099 & 0.151 & 0.150 & 0.124 & 0.096 \\
\hline & & 000 & 001 & 010 & 011 & 100 & 101 & 110 & 111 \\
\hline \multirow{9}{*}{ (b) } & 000 & 0.390 & 0.133 & 0.158 & 0.040 & 0.186 & 0.043 & 0.043 & 0.006 \\
\hline & 001 & 0.042 & 0.478 & 0.023 & 0.150 & 0.027 & 0.213 & 0.006 & 0.062 \\
\hline & 010 & 0.035 & 0.017 & 0.495 & 0.161 & 0.024 & 0.006 & 0.206 & 0.057 \\
\hline & 011 & 0.009 & 0.073 & 0.074 & 0.496 & 0.005 & 0.038 & 0.040 & 0.265 \\
\hline & 100 & 0.026 & 0.013 & 0.017 & 0.006 & 0.546 & 0.165 & 0.178 & 0.049 \\
\hline & 101 & 0.008 & 0.046 & 0.005 & 0.027 & 0.084 & 0.561 & 0.034 & 0.234 \\
\hline & 110 & 0.008 & 0.005 & 0.057 & 0.025 & 0.079 & 0.030 & 0.576 & 0.220 \\
\hline & 111 & 0.006 & 0.015 & 0.018 & 0.088 & 0.023 & 0.114 & 0.125 & 0.612 \\
\hline & $\pi$ & 0.021 & 0.050 & 0.062 & 0.110 & 0.095 & 0.166 & 0.187 & 0.310 \\
\hline
\end{tabular}

best solution found over time, a stationary probability close to one could be distributed on the optimal solution of the given problem instance.

EXAMPLE 5: Stationary probability distributions caused by different ESWO-II operators.

Table 6 shows two transition matrices of using different combinations of selection, mutation, and construction operators (i.e., S_reve $\times$ M_10 $\times$ C_L1 and S $\times$ M_05 $\times$ C_L2). We can see that the two matrices are not symmetric and are very different. In particular, their stationary distribution vectors $\pi$ differ significantly, which is in line with Corollary 2.

A significant observation arising from Table 6(b) arises from comparing the states 011 and 100. The state 100 has the higher fitness, but has a lower probability. As discussed in the introduction, this is an unusual case. It is quite different from simulated annealing, where, essentially by construction, the probability of a state depends only on its fitness, and in a monotonic fashion (as given by the standard Metropolis ideas). It will need further investigation but it seems reasonable that this could be because 011 is just one mutation from the optimal 111, and 100 is two mutations, hence further away and so likely to have a lower probability. This also makes sense because the analysis and selection together do not use the full fitness function but are trying instead to maximize the number of ones. Hence, they are, in a sense, working towards a simplified fitness function, which sometimes makes mistakes in that it takes 011 to be fitter than 100 . This alternative fitness that is effectively used by the selector seems to have some effect on the final distribution. We also note that the process is not like simulated annealing in which moves can be rejected on the basis of the fitness achieved; in ESWO all mutation 
Table 7: Stationary distributions: full strategy combinations (* denotes optimal solutions).

\begin{tabular}{|c|c|c|c|c|c|c|c|c|c|c|}
\hline No. & Transition matrix $\mathbf{P}$ & 000 & 001 & 010 & 011 & 100 & 101 & 110 & $111^{*}$ & Class \\
\hline 1 & & & & & נות & & U & 97 & & \\
\hline 2 & nd & 75 & 43 & 36 & 01 & 143 & 125 & 101 & & \\
\hline 3 & M_20 $\times$ C_rand & 162 & 139 & 133 & 107 & 139 & 0.124 & 0.107 & 37 & oor \\
\hline 4 & $\times \mathbf{M} \_05 \times$ C_LL1 $_{-}$ & 113 & 135 & 136 & 0.096 & 0.154 & 0.155 & 0.122 & 90 & boor \\
\hline 5 & S_re & 113 & 133 & 134 & 0.099 & 0.151 & 0.150 & 0.124 & 096 & boor \\
\hline 6 & S_re & 114 & 131 & 131 & 105 & 0.146 & 0.143 & .125 & 104 & boor \\
\hline 7 & S_rar & 125 & 125 & 125 & 125 & 0.125 & 0.125 & .125 & 25 & veras \\
\hline 8 & S_rar & 125 & 125 & 0.125 & 0.125 & 0.125 & 0.125 & 0.125 & 125 & verage \\
\hline 9 & S_rar & 0.125 & 0.125 & 0.125 & 0.125 & 0.125 & 0.125 & 0.125 & 0.125 & erage \\
\hline 10 & S_rev & 0.057 & 0.095 & 0.113 & 0.096 & 0.168 & 0.185 & 0.159 & 0.125 & rage \\
\hline 11 & S_rev & 0.056 & 0.093 & 0.111 & 0.096 & 0.164 & 0.180 & 0.161 & 0.134 & \\
\hline 12 & S_rev & 0.056 & 0.091 & 0.109 & 0.105 & 0.158 & 0.172 & 0.163 & 0.145 & \\
\hline 13 & S_rar & 0.087 & 0.113 & 0.116 & 0.119 & 0.133 & 0.141 & 0.144 & 0.148 & \\
\hline 14 & S_ra & 0.079 & 0.109 & 0.113 & 0.117 & 0.135 & 0.145 & 0.149 & 0.155 & 410 \\
\hline 15 & S_rar & 0.075 & 0.106 & 0.111 & 0.112 & 0.135 & 0.147 & 0.152 & 59 & arun \\
\hline 16 & $\mathbf{S} \times \mathbf{M}$ & 0.083 & 0.107 & 0.107 & 0.139 & 0.107 & 0.139 & 0.139 & 0.180 & ediun \\
\hline 17 & M_20 $\times$ C_L2 & 0.044 & 0.076 & 0.093 & 0.111 & 0.138 & 0.161 & 0.179 & 0.198 & liun \\
\hline 18 & $\mathbf{S} \times \mathbf{M} \_10 \times$ C_rand & 0.072 & 0.101 & 0.101 & 0.142 & 0.101 & 0.142 & 0.142 & 0.198 & medium \\
\hline 19 & S_rand $\times$ M_10 $\times$ C_L2 & 0.039 & 0.073 & 0.090 & 0.109 & 0.139 & 0.163 & 0.183 & 0.204 & good \\
\hline 20 & S_rand $\times$ M_05 $\times$ C_L2 & 0.038 & 0.072 & 0.089 & 0.107 & 0.139 & 0.165 & 0.184 & 0.206 & good \\
\hline 21 & $\mathbf{S} \times \mathbf{M} \_05 \times$ C_rand & 0.065 & 0.096 & 0.096 & 0.144 & 0.098 & 0.144 & 0.144 & 0.210 & good \\
\hline 22 & $\mathbf{S} \times \mathbf{M} \_20 \times \mathbf{C}_{-}$L1 & 0.059 & 0.094 & 0.096 & 0.127 & 0.110 & 0.150 & 0.154 & 0.211 & good \\
\hline 23 & $\mathbf{S} \times \mathbf{M} \_10 \times \mathbf{C}_{-}$L1 & 0.046 & 0.085 & 0.087 & 0.126 & 0.103 & 0.154 & 0.160 & 0.239 & good \\
\hline 24 & $S \times 1$ & 0.040 & 0.080 & 0.082 & 0.125 & 0.098 & 0.157 & 0.163 & 0.256 & better \\
\hline 25 & $S \times 1$ & 0 & 0.061 & 0.074 & 0.113 & 0.108 & 0.163 & 0.183 & 68 & \\
\hline 26 & $S \times 1$ & & & 0.0 & 0.111 & 0.100 & 0.165 & 0.186 & 0.295 & better \\
\hline 27 & $\mathbf{S} \times \mathbf{M} \_05 \times \mathbf{C} \_$L2 & 0.021 & 0.050 & 0.062 & 0.110 & 0.095 & 0.166 & 0.187 & 0.310 & best \\
\hline
\end{tabular}

moves are accepted. In fact, this supports our original motivations that understanding ESWO will need a good understanding of the separate stages, and their interaction.

Next, we use the following example to verify experimentally that certain strategies do make the optimal solution have higher $\pi_{i}$ values.

EXAMPLE 6: Key factors affecting the stationary probability distribution.

Consider further the previous examples where $f\left(x^{(j)}\right)=4 x_{1}+2 x_{2}+x_{3}+1$ is used. Then the optimal solution of the toy problem is 111, with fitness values increasing as we move to the right in the tables. Table 7 shows the stationary distribution vectors $\pi$ of transition matrices obtained by a full combination of selection, mutation, and construction strategies. The stationary probabilities of the optimal solution are displayed in the second to last column in ascending order, and indicative class values (i.e., poor, average, medium, good, better, or best) are displayed as a rough guide in the last column.

We can see from Table 7 as long as a randomized selection and a randomized construction are used together, the vector $\pi$ will be equally distributed with a probability 0.125 for each state. Thus, $\pi_{(111)}=0.125$ is the threshold value to judge if an implementation of the algorithm is efficient or not. Also, as long as an S_reve function is used, 
$\pi_{(111)}$ are no higher than average unless a C_L2 is jointly used, which makes $\pi_{(111)}$ a little bit higher than average on two cases (but still on the bottom of the medium list). Furthermore, as long as an S_rand or a C_rand operator (but not both) is used, $\pi_{(111)}$ values fall into the class medium or the bottom of class good. The interesting thing is that a joint use of $\mathbf{S}$ and $C_{-}$L2 achieves the top three $\pi_{(111)}$ values, and in particular, the smallest mutation rate (i.e., M_05) generates the highest value. The above observations suggest that, to achieve the best system performance, selection may play the most important role, construction the second, and mutation the third.

Observe that line 21 of Table 7 shows that a biased selector in itself is enough to cause a bias toward the optimal, even with an unbiased (random) constructor. This makes sense because if we count 0 as a flaw, then even with a random constructor, it has a $50 \%$ chance of being converted to 1 , and this will be an improvement. Arguably, overall, this makes ESWO-II rather more similar to standard iterative repair (e.g., see Minton et al., 1992) than to SWO.

\section{Conclusions}

In this paper, we have developed a formal framework for extending ESWO to ESWO-II by revising the ESWO's construction step to enable probabilistic choices among different possible destination states in a flexible way. Firstly, we focused on ESWO-II's convergence behavior. By a finite Markov chain analysis, we confirm that (unsurprisingly) although the global optimum is reachable after finite transitions with probability one, convergence to the global optimum is not. Also, after studying its transition matrix, we find that for the ESWO-II algorithm, the stationary distribution on individual states is not only affected but also controllable by the choices of the selection function, the mutation rate, and the construction function. The last result suggests that, by a proper implementation of the ESWO-II algorithm, even without using the strategy of saving the previous best solutions, if desired, the algorithm could be controlled so as to converge to an optimal solution (in the same sense as SA does when the temperature is cooled correctly).

Possibly, though further study is required, controlling such concentration around the optima will mean that an optimal solution will be reached much earlier than other solutions during the progress of the algorithm. Note that in simulated annealing, very small temperatures are best concentrated around optima, but are not the best for reducing the time to reach the optima, because the high concentration actually causes a loss of diversity in the search and so hitting times increase. With SA, this means that temperatures need to be carefully controlled. It is far from clear whether or not the same issues also apply to ESWO-II, and such an analysis would be a major goal of future work.

Major motivations for this study are that ESWO is a multistage algorithm and passes through partial assignments and so is rather different from SA. In particular, these properties make it novel for a Markov chain analysis. We believe the empirical results presented here also justify our interest in analyzing such a multistage algorithm. In particular, they show that the selection and construction stages can show rather different biases toward good solutions. It seems that the locality of the analysis and selection stages means that they work effectively using a slightly different fitness function than the original. This difference was revealed by nonmonotonic behavior in the overall probability distributions, and that would not occur in simulated annealing. In future work, we intend to further study this phenomenon. 
Finally, in our empirical studies, we considered time-independent operators. However, this is not forced; for example, for the mutation operator, we could use a (slowly)-varying mutation rate, which makes the corresponding Markov chain (quasi-) inhomogeneous. These advanced strategies will result in different Markov modeling and convergence behaviors, and we intend to investigate them in future.

\section{Acknowledgments}

The research described in this paper was funded by the Engineering and Physical Sciences Research Council (EPSRC), under grants EP/D061571/1 and EP/F033214/1.

\section{References}

Agapie, A. (1998). Genetic algorithms: Minimal conditions for convergence. Artificial Evolution, Lecture Notes in Computer Science, 1363:181-193.

Aickelin, U., Burke, E. K., and Li, J. (2009). An evolutionary squeaky wheel optimisation approach to personnel scheduling. IEEE Transactions on Evolutionary Computation, 13:433-443.

Aickelin, U., and Li, J. (2007). An estimation of distribution algorithm for nurse scheduling. Annals of Operations Research, 155:289-309.

Barbulescu, L., Watson, J. P., Whitley, L., and Howe, A. E. (2004). Scheduling space-ground communications for the air force satellite control network. Journal of Scheduling, 27:7-34.

Burke, E. K., Hyde, M., and Kendall, G. (2010). A squeaky wheel optimisation methodology for two dimensional strip packing. Computers \& Operations Research, 14:942-958.

Burke, E. K., and Newall, J. P. (2004). Solving examination timetabling problems through adaptation of heuristic orderings. Annals of Operations Research, 129:107-134.

Dorigo, M., Maniezzo, V., and Colorni, A. (1996). Ant system: Optimization by a colony of cooperating agents. IEEE Transactions on Systems, Man, and Cybernetics, Part B, 26:29-41.

Feng, G., and Lau, H. (2008). Efficient algorithms for machine scheduling problems with earliness and tardiness penalties. Annals of Operations Research, 159:83-95.

Feo, A., and Resende, M. G. C. (1989). A probabilistic heuristic for a computationally difficult set covering problem. Operations Research Letters, 8:67-71.

Fu, Z., Li, Y., Lim, A., and Rodrigues, B. (2007). Port space allocation with a time dimension. Journal of the Operational Research Society, 58:797-807.

Gendreau, M., and Potvin, J. Y. (2010). Handbook of meta-heuristics, 2nd ed. Berlin: Springer.

Ginsberg, M. L. (2001). Gib: Imperfect information in a computationally challenging game. Journal of Artificial Intelligence Research, 14:303-358.

Glover, F. (1989). Tabu search, Part I. ORSA Journal on Computing, 1:190-206.

Goldberg, D. (1989). Genetic algorithms in search, optimization and machine learning. Reading, MA: Addison-Wesley.

Hansen, P., and Mladenović, N. (1999). Variable neighbourhood search: Principles and applications. European Journal of Operational Research, 130:449-467.

Hoos, H. H., and Stutzle, T. (2005). Stochastic local search: Foundations and applications. San Mateo, CA: Morgan Kaufmann. 
Joslin, D., and Clements, D. P. (1999). Squeaky wheel optimization. Journal of Artificial Intelligence Research, 10:353-373.

Kirkpatrick, S., Gelatt, C., and Vecchi, M. (1983). Optimization by simulated annealing. Science, 220:671-680.

Koralov, L. B., and Sinai, Y. G. (2007). Theory of probability and random processes. Berlin: Springer.

Larrañaga, P., and Lozano, J. A. (2002). Estimation of distribution algorithms: A new tool for evolutionary computation. Dordrecht, The Netherlands: Kluwer Academic Publishers.

Li, J., and Kwan, R. S. K. (2003). A fuzzy genetic algorithm for driver scheduling. European Journal of Operational Research, 147:334-344.

Lim, A., Rodrigues, B., Xiao, F., and Zhu, Y. (2004). Crane scheduling with spatial constraints. Naval Research Logistics, 51:386-406.

Malaguti, E., and Toth, P. (2008). An evolutionary approach for bandwidth multicoloring problems. European Journal of Operational Research, 189:638-651.

Michiels, W., Aarts, E., and Korst, E. J. (2007). Theoretical aspects of local search. Berlin: Springer.

Minton, S., Johnston, M. D., Philips, A. B., and Laird, P. (1992). Minimizing conflicts: A heuristic repair method for constraint satisfaction and scheduling problems. Artificial Intelligence Journal, 58:161-205.

Papadimitriou, C. H., and Steiglitz, K. (1982). Combinatorial optimization-Algorithms and complexity. New York: Dover.

Rudolph, G. (1994). Convergence analysis of canonical genetic algorithms. IEEE Transactions on Neural Networks, 5:96-101.

Tijms, H. C. (2003). A first course in stochastic models. New York: Wiley. 
Copyright of Evolutionary Computation is the property of MIT Press and its content may not be copied or emailed to multiple sites or posted to a listserv without the copyright holder's express written permission. However, users may print, download, or email articles for individual use. 